\title{
The Korean version of the FRAIL scale: clinical feasibility and validity of assessing the frailty status of Korean elderly
}

Hee-Won Jung ${ }^{1,2}$, Hyun-Jung Yoo ${ }^{1}$, Si-Young Park ${ }^{1}$, Sun-Wook Kim ${ }^{1,2}$, Jung-Yeon Choi ${ }^{1}$, Sol-Ji Yoon ${ }^{1}$, Cheol-Ho Kim ${ }^{1,2}$, and Kwang-il Kim ${ }^{1,2}$

${ }^{1}$ Geriatric Center, Seoul National University Bundang Hospital, Seongnam; ${ }^{2}$ Department of Internal Medicine, Seoul National University College of Medicine, Seoul, Korea

Received: October 30, 2014 Revised : December 20, 2014 Accepted: January 6, 2015

\section{Correspondence to}

Kwang-il Kim, M.D.

Division of Geriatrics, Department of Internal Medicine, Seoul National University Bundang Hospital, 82 Gumi-ro 173beon-gil, Bundang-gu, Seongnam 13620, Korea

Tel: +82-31-787-7032

Fax: +82-31-787-4052

E-mail: kikim907@snu.ac.kr
Background/Aims: The fatigue, resistance, ambulation, illnesses, and loss of weight (FRAIL) scale is a screening tool for frailty status using a simple 5-item questionnaire. The aim of this study was to evaluate the clinical feasibility and validity of the Korean version of the FRAIL (K-FRAIL) scale.

Methods: Questionnaire items were translated and administered to 103 patients aged $\geq 65$ years who underwent a comprehensive geriatric assessment at the Seoul National University Bundang Hospital. In this cross-sectional study, the K-FRAIL scale was compared with the domains and the multidimensional frailty index of the comprehensive geriatric assessment. We also assessed the time required to complete the scale.

Results: The participants' mean age was 76.8 years (standard deviation [SD], 6.1), and 55 (53.4\%) were males. The mean overall frailty index was 0.19 (SD, 0.17). For K-FRAIL-robust, prefrail, and frail patients, the mean frailty indices were 0.09 , 0.18 , and 0.34 , respectively ( $p$ for trend $<0.001$ ). A higher degree of impairment in the K-FRAIL scale was associated with worse nutritional status, poor physical performance, functional dependence, and polypharmacy. The number of items with impairment in the K-FRAIL scale was positively associated with the frailty index $(\mathrm{B}=3.73, p<0.001)$. The K-FRAIL scale could differentiate vulnerability from robustness with a sensitivity of 0.90 and a specificity of 0.33 . Of all patients, 75 (72.8\%) completed the K-FRAIL scale within < 3 minutes.

Conclusions: The K-FRAIL scale is correlated with the frailty index and is a simple tool to screen for frailty in a clinical setting.

Keywords: Elderly; Frail; Geriatric assessment; Diagnosis

\section{INTRODUCTION}

Frailty is defined by a state of increased vulnerability to possible stressors caused by a decreased homeostatic reserve in older adults $[1,2]$. It is closely associated with clinical adverse outcomes including increased mortality and functional dependence, and thereby results in in- creased medical expenditure. Considering the rapid demographical aging in Korea, the issue of frailty in older adults is currently more important than ever [3].

Frailty has been well-recognized as a risk factor for adverse outcomes in patients undergoing medical treatment [4] or surgical procedures [5]. Moreover, knowing the frailty status of a patient can change the therapeutic 
goal [6] or modality [7] in the treatment of various diseases. Hence, an appropriate methodology to detect frailty is crucial, and many indices and criteria have been developed and evaluated in geriatric research.

Historically, various methods and concepts to define frailty have been developed and validated, from single gait speed $[8,9]$ to the frailty index (FI), which is calculated using more than 70 variables obtained in a comprehensive geriatric assessment (CGA) [10]. However, a brief screening tool, requiring the least amount of resource possible, to determine frailty status is necessary to facilitate the inclusion of frailty status in clinical research targeting the elderly population or as a screening tool in busy clinical practices.

Morley et al. [11] have suggested the fatigue, resistance, ambulation, illnesses, and loss of weight (FRAIL) scale, which includes a simple 5 -item questionnaire and has no requisite for physical examination, as an easy screening method for frailty status. The clinical validity of this scale has been reported, and the items of the scale have been shown to be associated with frailty biomarkers [11]. The scale has also been validated in the Asian population [12] and has been shown to predict mortality and disability similar to the FI determined using a higher number of variables [13]. Considering the advantages of this scale, we aimed to develop a Korean version of the FRAIL (K-FRAIL) scale and to evaluate its clinical feasibility and validity.

\section{METHODS}

\section{Study participants}

We prospectively included 103 patients aged $\geq 65$ years who underwent a CGA at an outpatient clinic and ward of the Seoul National University Bundang Hospital (SNUBH) between July 2014 and August 2014 in this study. Inclusion criteria were: (1) verbal communication via patients or guardians who were closely involved in the care of patients was possible; and (2) verbal consent for the study was provided. Apparently terminal patients with an expected survival of $<3$ months or patients staying in the emergency department were excluded. Because the study only involved a 5-item questionnaire that was administered additionally with the CGA, the potential harm of the study was considered to be min- imal. Therefore, the Institutional Review Board of the SNUBH approved the study only requiring verbal consent from patients or guardians.

\section{Measurements}

The CGA at the SNUBH was performed by two experienced nurses specialized in geriatric assessment. Domains of the CGA included activities of daily living (ADL; range, o to 100; decreasingly worse) using the modified Barthel index [14], instrumental activities of daily living (IADL; range, o to 5 for males; range, o to 8 for females, decreasingly worse) using the Lawton and Brody index [15], comorbidity status using the Charlson comorbidity index (increasingly worse) [16], mood using the Korean version of the geriatric depression scale short form (GDS-SF; range, o to 15; increasingly worse) [17], cognition using the Korean mini-mental state examination (K-MMSE; range, o to 30; decreasingly worse) [18], nutritional status using the mini nutritional assessment (MNA; range, o to 30; decreasingly worse) [19], and physical performance using the timed up and go test (TUGT) [20]. For dichotomized comparisons, cut-off values were set at $\leq 99$ for the modified Barthel index for ADL dependency, $\leq 4$ for males and $\leq 7$ for females for the Lawton and Brody index for IADL dependency, $\leq 24$ for the K-MMSE (cognitive impairment), $\geq 20$ for the TUGT or mobility limitation when the patient was unable to perform the TUGT, $\geq 9$ for the GDS-SF (depressive mood), and $\leq 23.5$ for the MNA (risk of malnutrition). Data for current and previous body weight, serum albumin level, and hemoglobin level were also collected.

The multidimensional FI was calculated from the CGA data using weighting factors for each domain based on previously published equations [3]. For the FI, we used previously published cut-off values of $\geq 0.20$ for prefrail and $\geq 0.35$ for frail. The FI used in our study has been shown to be significantly correlated with cardiovascular health study (CHS) frailty criteria, and has also been shown to have an acceptable predictive capability for mortality and functional decline in a previous publication [3].

The K-FRAIL scale, based on the original FRAIL scale published by Morley et al. [11], was translated into Korean. The questionnaire was translated by HWJ and reviewed by HJY, SYP, and SWK. A final version of the translated scale is shown in Supplementary Fig. 1. In this 
questionnaire, a score of o was considered as robust, 1 to 2 as prefrail, and 3 to 5 as frail. This questionnaire was administered during the CGA session with assistance of nurse, and time taken to complete the questionnaire was recorded as following; $<3,3$ to 5,5 to 10 , and $\geq 10$ minutes.

\section{Statistical analysis}

To compare variables for baseline characteristics by frailty status derived from the K-FRAIL scale, we used the Kruskal-Wallis test for continuous variables and the chisquare test for discrete variables. Differences in the FI from robust to frail by the K-FRAIL scale were evaluated by the Kruskal-Wallis test with a post hoc analysis using the Mann-Whitney $U$ test. Sensitivity, specificity, positive predictive value, and negative predictive value of the K-FRAIL scale for differentiating robustness from vulnerability (prefrail and frail) were calculated. The associations of the CGA domains with each item of the K-FRAIL scale were evaluated by ordinary least square analysis. A two-tailed $p$ value of $<0.05$ was considered statistically significant. Statistical analyses were per- formed using PASW version 18.0 (SPSS Inc., Chicago, IL, USA).

\section{RESULTS}

\section{Participant characteristics}

The participants had a mean age of 76.8 years (standard deviation [SD], 6.1), and 55 (53.4\%) were males. Of all patients, 80 (77.7\%) were ADL independent and 83 (80.5\%) IADL independent. Moreover, 67 patients (65.0\%) were taking 5 or more medications regularly, indicating polypharmacy. The mean FI was 0.19 (SD, 0.17).

Among the 103 participants, 25(24.3\%) were determined as robust, $60(58.3 \%)$ as prefrail, and $18(17.5 \%)$ as frail by the K-FRAIL scale. The numbers of patients by K-FRAIL score were 25 with a score of o (24.3\%), 36 with a score of $1(35.0 \%), 24$ with a score of $2(23.3 \%), 12$ with a score of $3(11.7 \%), 5$ with a score of $4(4.9 \%)$, and 1 with a score of 5 (1.0\%). The prevalence rates for positivity of each item in the K-FRAIL scale were 38 for fatigue (36.9\%), 40 for resistance (38.8\%), 27 for ambulation (26.2\%), 3 for illness

Table 1. Baseline characteristics of participants by frailty status assessed by the K-FRAIL scale

\begin{tabular}{|c|c|c|c|c|}
\hline Variable & Robust $(\mathrm{n}=25)$ & Prefrail $(\mathrm{n}=60)$ & Frail $(\mathrm{n}=18)$ & $p$ value \\
\hline Age, yr & $75 \cdot 6 \pm 5 \cdot 9$ & $76.6 \pm 6.0$ & $79.1 \pm 6.4$ & 0.212 \\
\hline Male sex & $13(52.0)$ & $28(46.7)$ & $7(38.9)$ & $0.723^{\mathrm{a}}$ \\
\hline ADL (modified Barthel index) & $100.0 \pm 0.0$ & $98.3 \pm 5.5$ & $92.5 \pm 11.1$ & $<0.001$ \\
\hline \multicolumn{5}{|l|}{ IADL (Lawton and Brody index) } \\
\hline Male & $4.9 \pm 0.3$ & $4.8 \pm 0.6$ & $4.7 \pm 0.5$ & 0.398 \\
\hline Female & $8.0 \pm 0.0$ & $7 \cdot 3 \pm 1.4$ & $6.1 \pm 2.0$ & 0.008 \\
\hline Cognition (K-MMSE score) & $25.1 \pm 3.5$ & $23.3 \pm 4.8$ & $20.8 \pm 4.8$ & 0.017 \\
\hline Mood (K-GDS SF score) & $2.8 \pm 3.1$ & $5 \cdot 1 \pm 4 \cdot 1$ & $7 \cdot 3 \pm 3.1$ & $<0.001$ \\
\hline Nutrition (MNA score) & $25 \cdot 6 \pm 3.0$ & $5.1 \pm 4.1$ & $19.0 \pm 4.0$ & $<0.001$ \\
\hline No. of medications & $5 \cdot 4 \pm 3 \cdot 7$ & $6.4 \pm 4.4$ & $9.0 \pm 4 \cdot 3$ & 0.014 \\
\hline Comorbidity (Charlson comorbidity index) & $2.8 \pm 2.0$ & $2.8 \pm 2.4$ & $3.2 \pm 2.4$ & 0.568 \\
\hline Physical performance, sec $\left(T^{\prime} U_{T}{ }^{b}\right)$ & $13 \cdot 5 \pm 3.0$ & $15 \cdot 3 \pm 6.2$ & $24.0 \pm 14.1$ & 0.007 \\
\hline Serum albumin, g/dL & $4.0 \pm 0.4$ & $3.9 \pm 0.5$ & $3.6 \pm 0.5$ & 0.010 \\
\hline Frailty index & $0.09 \pm 0.08$ & $0.18 \pm 0.16$ & $0.34 \pm 0.19$ & $<0.001$ \\
\hline
\end{tabular}

Values are presented as mean \pm SD or number $(\%)$.

K-FRAIL, Korean version of the fatigue, resistance, ambulation, illnesses, and loss of weight; ADL, activities of daily living; IADL, instrumental activities of daily living; K-MMSE, Korean mini-mental state examination; K-GDS SF, Korean version of the geriatric depression scale short form; MNA, mini nutritional assessment; TUGT, timed up and go test.

${ }^{a}$ A $p$ value from chi-square test.

${ }^{\mathrm{b}}$ Value available for 25 robust, 50 prefrail, and 13 frail participants. 


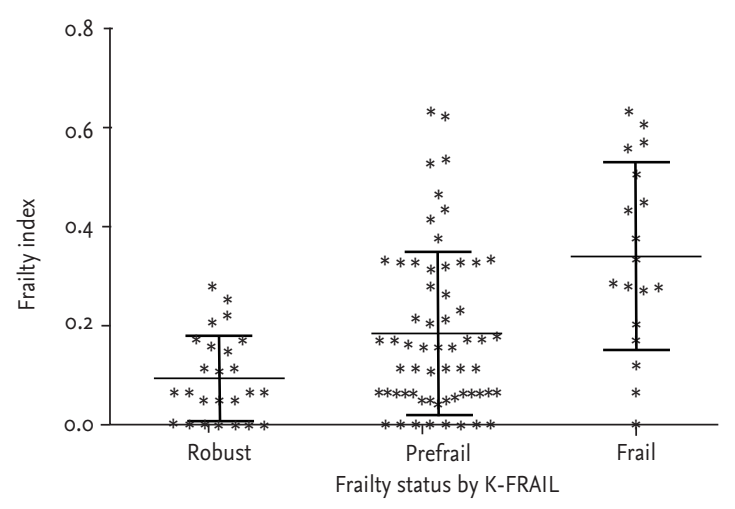

Figure 1. Distribution of the frailty index by frailty status assessed by the Korean version of the fatigue, resistance, ambulation, illnesses, and loss of weight (K-FRAIL) scale.

\section{(2.9\%), and 37 for loss of weight (35.9\%).}

Baseline demographic and functional, physical, and laboratory characteristics grouped by frailty status using the K-FRAIL scale are shown in Table 1. ADL, cognition, mood, nutritional status, and physical performance were significantly different by frailty status as determined by the Kruskal-Wallis test. A difference in IADL was only observed in females. In contrast, age, sex, and comorbidity status were not significantly different by frailty status based on the K-FRAIL scale.

\section{Correlation between the frailty index and the K-FRAIL scale}

The FI was calculated using domains of physical performance, ADL, IADL, cognition, and serum albumin levels. The mean FI was 0.09 for K-FRAIL-robust, 0.18 for prefrail, and 0.34 for frail patients $(p<0.001$ by Kruskal-Wallis test) (Fig. 1). The post hoc analysis using the Mann-Whitney $U$ test showed $p=0.017$ for robust and prefrail, $p=0.002$ for prefrail and frail, and $p<0.001$ for robust and frail. The number of impaired items in the K-FRAIL scale (range, o to 5) was positively associated with the FI (B = 3.73; standard error [SE], 0.57; $p<0.001)$, as calculated by ordinary least squares regression analysis.

Using FI as a standard assessment for frailty status, a performance analysis of the K-FRAIL scale as a screening test was performed. For differentiating vulnerability (prefrail and frail) from robustness, the sensitivity and specificity of the K-FRAIL scale were 0.90 (95\% confidence interval [CI], 0.76 to 0.97 ) and 0.33 (95\% CI, 0.22 to 0.46), respectively. The positive likelihood ratio was 1.35, the negative likelihood ratio 0.30 , the positive predictive value 0.46 , and the negative predictive value 0.84 .

\section{Correlation between items in the K-FRAIL scale and domains of the comprehensive geriatric assessment} Using frailty status by the K-FRAIL scale as a linear term, a trend of incremental impairments in ADL $(p<0.001)$, $\operatorname{mood}(p=0.004)$, nutritional status $(p=0.007)$, and physical performance $(p=0.039)$ with increasing frailty status was found. IADL was incrementally worse in women with increasing frailty status $(p=0.002)$; however, this association was not significant in men $(p=0.386)$. These incremental relationships were not observed for the cognitive domain $(p=0.963)$, comorbidity status $(p=$ 0.759), and serum albumin levels ( $p=0.157)$.

Furthermore, each item of the K-FRAIL scale was assessed for its association with the functional domains of the CGA (Table 2). Using ordinary least square analysis, we found that the fatigue and resistance items were positively associated with depressive mood. The resistance and ambulation items were positively associated with ADL and IADL impairment, cognitive impairment, and mobility limitation, and loss of weight was associated with mobility limitation and risk of malnutrition $(\mathrm{B}=$ 0.34; SE, 0.09; $p<0.001)$. The illness item was not associated with any of the functional domains.

\section{Clinical feasibility}

No adverse events from administering the K-FRAIL scale were noted during the CGA. The two nurses of the geriatric division reported no specific difficultness in adapting the K-FRAIL scale to their clinical setting. In 75 participants (72.8\%), completing the K-FRAIL scale with assistance of a nurse took $<3$ minutes. Among all patients, 24 participants (23.3\%) needed 3 to 5 minutes, and four participants $(3.9 \%) 5$ to 10 minutes for completing the K-FRAIL scale. The time required to complete the scale was associated with frailty status as assessed by the K-FRAIL scale ( $p$ for trend < 0.001).

\section{DISCUSSION}

In this prospective study, the K-FRAIL scale was associated with domains of the CGA and with the multidimensional FI in Korean elderly patients undergoing a CGA 


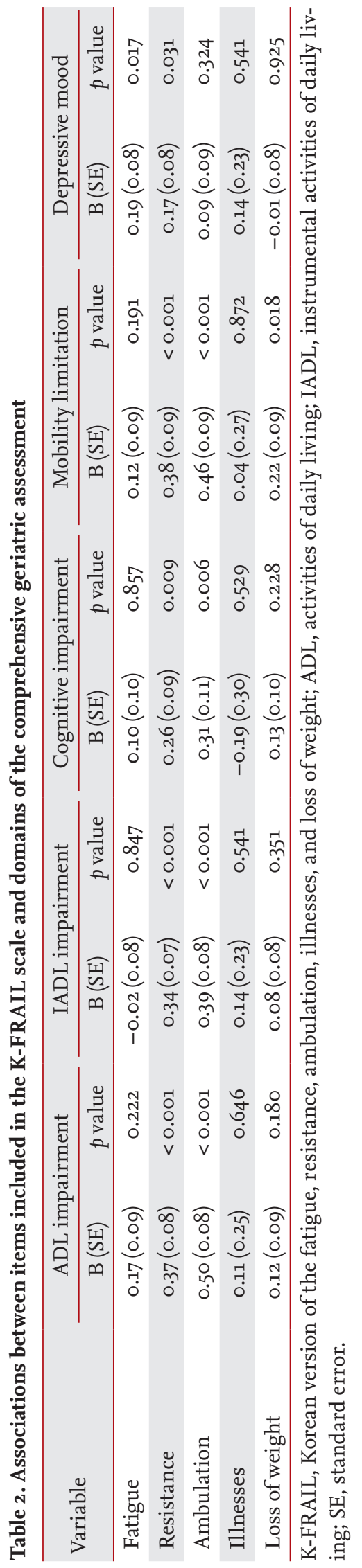

in a tertiary hospital. As a screening test, the K-FRAIL scale showed high sensitivity and negative predictive value in differentiating vulnerability from robustness. The questionnaire could be relatively easily and quickly administered. To the best of our knowledge, this is the first report on this frailty screening method in the Korean elderly population.

Although frailty is important for health outcomes, adapting the concept of frailty to clinical research or practice in Korea was with barriers for clinicians. Because physicians are typically only allowed to spend less than 3 to 5 minutes on each patient in most outpatient clinics, measuring the CHS frailty criteria by administering a questionnaire and testing grip strength and gait speed, or assessing the FI is not possible in most circumstances. Furthermore, only few hospitals in Korea have the resources to perform a CGA regularly and with dedicated personnel. In contrast, the K-FRAIL scale can be quickly completed by patients or guardians with the assistance of a nurse before the examination by a physician. Moreover, interpreting the results of this scale (robust vs. prefrail vs. frail) is easy for most clinicians. Therefore, the K-FRAIL scale can be a useful screening tool in most busy clinical practices in Korea.

Items in the K-FRAIL scale have distinct characteristics. The fatigue item is associated with depressive mood rather than physical performance. As shown in the original study by Morley et al. [11], the illness item had a very low prevalence for positivity and less effect on the composition of the K-FRAIL scale. This low weighing of comorbidity for a frailty scale is in accordance to original concept of physical frailty by Fried et al. [1], which suggested that frailty is an independent clinical feature from comorbidity or functional impairment. The loss of weight item can also be found in the CHS frailty criteria or the Study of Osteoporosis Fracture frailty criteria [21], and is associated with risk of malnutrition. In our study, the resistance and ambulation items were strongly associated with physical performance, ADL, and IADL impairment. Therefore, similar to the CHS frailty criteria, which include two physical performance items (grip strength and gait speed), the original FRAIL scale and the K-FRAIL scale focus on physical performance. Since body weight is already stabilized at low and fatigue might not be experienced by participants with a low activity level, this domain assignment in the FRAIL scale 
may explain the reason for the inclusion of some participants with a very high FI in the prefrail group in the K-FRAIL scale.

In this study, the FI (as assessed by the CGA) was distributed according to frailty status (as assessed by the K-FRAIL scale). Interestingly, the distribution of the FI in the prefrail and frail groups was lower than that in our previous study, in which we suggested a FI cut-off of 0.20 for prefrail and 0.35 for frail [3]. The distributions of the FIs for prefrail by the K-FRAIL scale were consistent with a clinical frailty scale in the Canadian study of health and aging [10] which showed a mean FI of 0.16 to 0.22 among vulnerable and 0.27 to 0.43 among frail individuals. Based on this observation, interpreting the multidimensional FI for categorized frailty status might be similarly performed according to original FI of accumulation of deficit model from Canadian study of health and aging [22].

Although this is one of the first prospective studies showing that a wide adaption of the concept of frailty in Korea is feasible, there are limitations in this study. The relatively small prospective study was conducted in single tertiary hospital with staff experienced in geriatric medicine. Therefore, the findings might not be generalizable to other settings. Hence, ongoing larger-scaled studies in different settings in Korea will be helpful for the generalizability. Because the K-FRAIL scale was measured on the same day as the CGA, answering bias might have occurred. However, since the FI was calculated after completion of the CGA, positive bias related to the association between frailty status by K-FRAIL and FI is less likely. Because of the study design, outcomes including functional decline and mortality could not be assessed in our study. Nevertheless, the K-FRAIL scale showed a strong correlation with the previously validated FI and multiple domains of the CGA, which are well known to predict adverse outcomes in elderly population.

In conclusion, the K-FRAIL scale can be easily measured and is associated with domains of the CGA and the previously validated FI in Korean elderly patients. Based on the results from our study, we suggest that the K-FRAIL can be used to screen for frailty in Korean older adults.

\section{KEY MESSAGE}

1. The Korean version of the fatigue, resistance, ambulation, illnesses, and loss of weight (K-FRAIL) scale is correlated with domains of the comprehensive geriatric assessment and the frailty index in Korean elderly patients.

2. The K-FRAIL scale can be easily measured with less than 3 minutes in most patients and with only few resources.

\section{Conflict of interest}

No potential conflict of interest relevant to this article was reported.

\section{REFERENCES}

1. Fried LP, Tangen CM, Walston J, et al. Frailty in older adults: evidence for a phenotype. J Gerontol A Biol Sci Med Sci 2001;56:M146-M156.

2. Clegg A, Young J, Iliffe S, Rikkert MO, Rockwood K. Frailty in elderly people. Lancet 2013;381:752-762.

3. Jung HW, Kim SW, Ahn S, et al. Prevalence and outcomes of frailty in Korean elderly population: comparisons of a multidimensional frailty index with two phenotype models. PLoS One 2014;9:e87958.

4. Extermann M, Hurria A. Comprehensive geriatric assessment for older patients with cancer. J Clin Oncol 2007;25:1824-1831.

5. Kim SW, Han HS, Jung HW, et al. Multidimensional frailty score for the prediction of postoperative mortality risk. JAMA Surg 2014;149:633-640.

6. American Diabetes Association. Standards of medical care in diabetes: 2014. Diabetes Care 2014;37 Suppl 1:S14-S80.

7. Stortecky S, Schoenenberger AW, Moser A, et al. Evaluation of multidimensional geriatric assessment as a predictor of mortality and cardiovascular events after transcatheter aortic valve implantation. JACC Cardiovasc Interv 2012;5:489-496.

8. Peel NM, Kuys SS, Klein K. Gait speed as a measure in geriatric assessment in clinical settings: a systematic review. J Gerontol A Biol Sci Med Sci 2013;68:39-46.

9. Studenski S, Perera S, Patel K, et al. Gait speed and surviv- 
al in older adults. JAMA 2011;305:50-58.

10. Rockwood K, Song X, MacKnight C, et al. A global clinical measure of fitness and frailty in elderly people. CMAJ 2005;173:489-495.

11. Morley JE, Malmstrom TK, Miller DK. A simple frailty questionnaire (FRAIL) predicts outcomes in middle aged African Americans. J Nutr Health Aging 2012;16:601-608.

12. Woo J, Leung J, Morley JE. Comparison of frailty indicators based on clinical phenotype and the multiple deficit approach in predicting mortality and physical limitation. J Am Geriatr Soc 2012;60:1478-1486.

13. Malmstrom TK, Miller DK, Morley JE. A comparison of four frailty models. J Am Geriatr Soc 2014;62:721-726.

14. Mahoney FI, Barthel DW. Functional evaluation: the barthel index. Md State Med J 1965;14:61-65.

15. Lawton MP, Brody EM. Assessment of older people: self-maintaining and instrumental activities of daily living. Gerontologist 1969;9:179-186.

16. Charlson ME, Pompei P, Ales KL, MacKenzie CR. A new method of classifying prognostic comorbidity in longitudinal studies: development and validation. J Chronic Dis
1987;40:373-383.

17. Burke WJ, Roccaforte WH, Wengel SP. The short form of the Geriatric Depression Scale: a comparison with the 30item form. J Geriatr Psychiatry Neurol 1991;4:173-178.

18. Kang Y, Na DL, Hahn S. A validity study on the Korean Mini-Mental State Examination (K-MMSE) in dementia patients. J Korean Neurol Assoc 1997;15:300-308.

19. Vellas B, Guigoz Y, Garry PJ, et al. The Mini Nutritional Assessment (MNA) and its use in grading the nutritional state of elderly patients. Nutrition 1999;15:116-122.

20. Podsiadlo D, Richardson S. The timed "Up \& Go": a test of basic functional mobility for frail elderly persons. J Am Geriatr Soc 1991;39:142-148.

21. Ensrud KE, Ewing SK, Taylor BC, et al. Comparison of 2 frailty indexes for prediction of falls, disability, fractures, and death in older women. Arch Intern Med 2008;168:382389.

22. Rockwood K, Mitnitski A. Frailty defined by deficit accumulation and geriatric medicine defined by frailty. Clin Geriatr Med 2011;27:17-26. 
Fatigue (피로) : 지난 한 달 동안 피곤하다고 느끼 적이 있습니까?

$1=$ 항상 그렇다 $2=$ 거의 대부분 그렇다 $3=$ 종종 그렇다 $4=$ 가끔씩 그렇다 $5=$ 전혀 그렇지 않다

$1=$ 항상 그렇다 $2=$ 거의 대부분 그렇다 $3=$ 종종
1,2 로 답변하면 점수는 1 점, 이외에는 0 점

Resistance (저항) : 도움이 없이 혼자서 쉬지 않고 10 개의 계단을 오르는데 힘이 듭니까?

예=1점, 아니오=0점

Ambulation (이동) : 도움이 없이 300미터를 혼자서 이동하는데 힘이 듭니까?

예=1점, 아니오 $=0$ 점

Illness (지병) : 의사에게 다음 질병이 있다고 들은 적이 있습니까?

(고혈압, 당뇨, 암, 만성 폐 질환, 심근 경색, 심 부전, 협심증, 천식, 관절염, 뇌경색, 신장 질환)

0-4개는 0점, 5-11개는 1점

Loss of weight (체중 감소) : 현재와 1년 전의 체중은 몇 $\mathrm{kg}$ 이었습니까?

1 년 간 $5 \%$ 이상 감소한 경우에 1 점, $5 \%$ 미만 감소한 경우에 0 점.

Supplementary Figure 1. The Korean version of the fatigue, resistance, ambulation, illnesses, and loss of weight (K-FRAIL) scale. A score of o indicates robust, 1 to 2 prefrail, and 3 to 5 frail. 